\title{
Utilização do Inventário de Habilidades Sociais no Diagnóstico do Transtorno de Ansiedade Social
}

\author{
Using the Social Skills Inventory in the Diagnosis of Social Anxiety Disorder
}

\author{
Antonio Paulo Angélico*, $a$, José Alexandre S. Crippa ${ }^{b} \&$ Sonia Regina Loureiro $^{b}$ \\ ${ }^{a}$ Universidade Federal de São João del-Rei, São João del-Rei, Brasil \\ $\&{ }^{b}$ Universidade de São Paulo, Ribeirão Preto, Brasil
}

\begin{abstract}
Resumo
Este estudo objetivou verificar as associações entre as manifestações clínicas e comportamentais do Transtorno de Ansiedade Social (TAS) e aferir a validade discriminativa do Inventário de Habilidades Sociais (IHS-Del-Prette) no diagnóstico deste transtorno. Participaram 1006 estudantes universitários, na faixa etária entre 17 e 35 anos, de ambos os gêneros. Posteriormente, 86 participantes foram randomicamente selecionados desta amostra inicial e agrupados como casos e não-casos de TAS por meio de avaliação clínica sistemática. Os resultados obtidos indicaram que quanto mais elaborado for o repertório de habilidades sociais de um indivíduo, menor será a sua probabilidade de satisfazer os critérios de rastreamento de indicadores diagnósticos para o TAS. Além disso, o IHS-Del-Prette demonstrou distinguir significativamente indivíduos com e sem TAS, evidenciando-se a sua validade discriminativa.

Palavras-chave: Transtorno de ansiedade social, habilidades sociais, validade, manifestações clínicas.

Abstract

This study aimed to assess the relationship between clinical and behavioral manifestations of the Social Anxiety Disorder (SAD) and verify the discriminative validity of the Social Skills Inventory (SSI-DelPrette) in the diagnosis of this disorder. The participants were 1,006 undergraduates, aged between 17 and 35 years old, both genders. Subsequently, 86 participants were randomly selected from the initial sample and grouped as SAD cases and non-SAD cases through systematic clinical evaluation. The results indicated that the more elaborate the repertoire of social skills of an individual is, the lower his/her likelihood of meeting the screening criteria of diagnostic indicators for SAD. Furthermore, the SSI-Del-Prette has demonstrated to significantly distinguish individuals with and without SAD, evidencing, thus, its discriminative validity.
\end{abstract}

Keywords: Social anxiety disorder, social skills, validity, clinical manifestations.

O Transtorno de Ansiedade Social (TAS), ou fobia social, é um transtorno psiquiátrico grave que acarreta sofrimento e perdas significativas de oportunidades para o seu portador. Conforme os critérios diagnósticos do DSM-IV (American Psychiatric Association [APA], 1994), indivíduos com TAS manifestam um medo excessivo, persistente e irracional de serem vistos comportando-se de um modo humilhante ou embaraçoso pela demonstração de ansiedade ou de desempenho inadequado - e de conseqüente desaprovação ou rejeição por parte dos outros.

\footnotetext{
"O artigo foi derivado de parte da Tese de Doutorado do $1^{\circ}$ autor, sob orientação do terceiro autor, apresentada no Programa de Pós-Graduação em Saúde Mental da Faculdade de Medicina de Ribeirão Preto da Universidade de São Paulo. Os autores agradecem à Coordenação de Aperfeiçoamento de Pessoal de Nível Superior (CAPES) pelo auxílio financeiro fornecido ao $1^{\circ}$ autor para o desenvolvimento da pesquisa (2005-2009).

Endereço para correspondência: Praça Dom Helvécio, 74, Dom Bosco, São João del-Rei, MG, Brasil 36301-160. E-mail: angelico@rnp.fmrp.usp.br, jcrippa@fmrp.usp.br e srlourei@fmrp.usp.br
}

Por esses critérios, fica subentendido que há dois subtipos de TAS: o generalizado (medo da maioria das situações de interação social e de desempenho) e o circunscrito, específico ou não-generalizado (medo de uma situação pública de desempenho e de algumas situações de interação social), cuja caracterização pode ser útil na predição do curso, prognóstico e na resposta ao tratamento. As situações de desempenho (ou circunstâncias de desempenho) mais temidas são falar em público, comer, beber, escrever e tocar um instrumento na frente dos outros, usar banheiro público e entrar em uma sala onde já se encontram pessoas sentadas. Falar em público, entretanto, é considerado o medo social mais prevalente (Furmark, 2000). As situações interacionais abrangem conversar ao telefone, falar com pessoas estranhas, participar de festas e reuniões sociais, interagir com o sexo oposto, conversações face-a-face com estranhos e autoridades e devolver mercadoria em uma loja (Hazen \& Stein, 1995, citados por Falcone \& Figueira, 2001; Furmark, 2000). O indivíduo com TAS, geralmente, evita tais situações ou as suporta com intensa ansiedade e sofrimento. 
Conforme os dados de vários estudos, as pessoas com TAS têm avaliado sua qualidade de vida como prejudicada, mostrando insatisfação com a vida e também com sua saúde (Safren, Heimberg, Brown, \& Holle, 1996-1997; Stein \& Kean, 2000; Wittchen \& Beloch, 1996; Wittchen, Fuetsch, Sonntag, Muller, \& Liebowitz, 2000). Neste sentido, a evitação fóbica apresentada pelos indivíduos com TAS frente às situações sociais pode limitar as suas possibilidades de sucesso profissional pela escolha de um trabalho menos qualificado, ou manter um relacionamento infeliz para não enfrentar a ansiedade de encontrar novas pessoas (Falcone \& Figueira, 2001). Este padrão de comportamento evitativo de situações sociais, característico do TAS, favorece limitações na vida profissional, acadêmica, social ou sexual da pessoa portadora de tal transtorno. Assim, esses apontamentos caracterizam a necessidade do diagnóstico precoce de tal transtorno e de modalidades de tratamento eficazes que atendem as necessidades sociais, pessoais e profissionais destas pessoas.

Dada a relevância das habilidades sociais nas manifestações clínicas do TAS, cada vez mais as medidas desse repertório vêm se constituindo em elemento importante na avaliação clínica deste transtorno. O déficit em habilidades sociais parece comum entre os fóbicos sociais, principalmente na forma generalizada (Falcone, 1998; Herbert, Hope, \& Bellack, 1992). Por outro lado, alguns autores argumentam que os fóbicos sociais possuem habilidades sociais em seu repertório, mas que sua exibição fica inibida durante a manifestação de ansiedade (Furmark, 2000; Heimberg, Dodge, \& Becker, 1987), caracterizando, assim, um déficit de fluência (Angélico, Crippa, \& Loureiro, 2006; Z. A. P. Del Prette $\&$ Del Prette, 2005).

Nesse contexto é que se inserem os instrumentos de avaliação das habilidades sociais. Há pouco mais de uma década, Z. A. P. Del Prette, Del Prette e Barreto (1998) assinalaram que, embora diversos instrumentos de medida tivessem sido construídos na área de estudo das habilidades sociais, poucos foram elaborados ou validados para o contexto cultural brasileiro. Ainda que existam aspectos do repertório de habilidades sociais comuns a diversas culturas, certas especificidades aconselham a adaptação de instrumentos elaborados em outros contextos ou, mesmo, a construção de novos instrumentos a partir de características próprias da subcultura para a qual se destinam.

Nessa época, alguns instrumentos já tinham sido aferidos para o contexto brasileiro. Uma escala para a avaliação da competência social de pacientes psiquiátricos, por meio do desempenho de papéis, foi elaborada por Bandeira e Tremblay (1998), com propriedades psicométricas satisfatórias (Bandeira, 2002). A Escala Brasileira para a Medida de Assertividade (Ayres, 1994) e a Escala de Assertividade de Rathus, adaptada e validada para o contexto brasileiro (Pasquali \& Gouveia, 1990), visam avaliar especificamente a assertividade e não as habilidades sociais consideradas como um conceito mais abrangente.
Para suprir a falta de uma medida que avaliasse as habilidades sociais em geral dos indivíduos, Z. A. P. Del Prette e Del Prette (2001) elaboraram e validaram o Inventário de Habilidades Sociais (IHS-Del-Prette), visando avaliar o repertório interpessoal de estudantes universitários. Trata-se de um instrumento de fácil aplicação que cobre um grande número de situações e comportamentos interpessoais.

No entanto, nenhum estudo anterior aferiu as propriedades psicométricas deste instrumento em amostras com TAS, sistematicamente avaliadas. Considera-se que, ainda que o déficit em habilidades sociais não esteja presente em muitos fóbicos sociais, é recomendável que a sua avaliação seja realizada, independentemente de manifestarem estes déficits, para efeitos tanto de caracterização do repertório desses indivíduos quanto para planejar programas de tratamento efetivos que atendam às suas demandas interpessoais e acadêmicas de modo mais direto e objetivo. Para tal, faz-se necessário demonstrar a validade das medidas comportamentais das habilidades sociais e suas associações a medidas clínicas do TAS, o que se configura como o proposto nesse estudo. Sendo assim, para atender essa lacuna na área, este estudo objetivou:

1.Verificar se as manifestações comportamentais do TAS, identificadas por meio do IHS-Del-Prette, estão associadas às manifestações clínicas, detectadas com a aplicação do Inventário de Fobia Social (SPIN), em estudantes universitários;

2. Avaliar a validade concorrente entre o IHS-Del-Prette e o Inventário de Fobia Social (SPIN);

3. Aferir a validade discriminativa e preditiva do IHSDel-Prette no diagnóstico do TAS;

4. Verificar o poder discriminativo dos itens que compõem o IHS-Del-Prette para rastreamento do TAS.

\section{Método}

Esta pesquisa constitui parte integrante de um estudo maior que visou a avaliação sistemática dos aspectos epidemiológicos, clínicos e comportamentais do TAS, por meio de instrumentos de auto e heteroavaliação, em uma população de estudantes universitários, independente do nível socioeconômico, matriculados em diversos cursos de duas instituições de ensino superior, uma pública (Universidade de São Paulo [USP], Brasil) e outra particular (Universidade de Franca [UNIFRAN], Brasil), ambas em cidades de médio porte do interior do estado de São Paulo. O estudo foi apreciado e aprovado pelo Comitê de Ética em Pesquisa da Faculdade de Medicina de Ribeirão Preto da USP (Proc. HCRP n ${ }^{\circ}$ 6064/2006).

\section{Participantes}

Inicialmente, foram contatados 1173 alunos, resultando após a aplicação dos critérios demográficos de inclusão em uma amostra de 1006 estudantes avaliados, na faixa etária entre 17 e 35 anos, de ambos os gêneros. 
Angélico, A. P., Crippa, J. A. S. \& Loureiro, S. R. (2012). Utilização do Inventário de Habilidades Sociais no Diagnóstico do Transtorno de Ansiedade Social.

Adotou-se como critério de exclusão o uso de neurolépticos. Dessa primeira amostra, foram randomicamente selecionados 86 participantes agrupados como casos $(n$ $=45)$ e não-casos de TAS $(n=41)$, por meio de avaliação clínica sistemática.

\section{Instrumentos}

Social Phobia Inventory (SPIN). Escala autoaplicável, caracterizando-se como um instrumento breve, composto por 17 itens, de fácil administração, e capaz de detectar sintomas de medo (itens 1, 3, 5, 10, 14 e 15), evitação (itens 4, 6, 8, 9, 11, 12 e 16) e fisiológicos (itens 2, 7, 13 e 17). Cada item pode ser classificado de 0 (nunca) até 4 (extremamente), em uma escala de 5 pontos, com escore total variando de 0 a 68 (Connor et al., 2000). Tal instrumento foi traduzido e adaptado para o português do Brasil por Osório (2008), apresentando boas qualidades psicométricas, com consistência interna de 0,90 e confiabilidade interavaliadores com índices entre 0,86 e 0,98.

Mini-SPIN. Versão abreviada do SPIN, composta por três itens $(6-$ evitar falar com pessoas por medo de embaraço; 9 - evitar ser o centro das atenções; e 15 - temer ficar envergonhado), avaliados em uma escala de cinco pontos. Usando um escore de corte igual ou maior que seis, a Mini-SPIN demonstrou possuir boas propriedades psicométricas: uma sensibilidade de $88,7 \%$, especificidade de $90 \%$, valor preditivo positivo de $52,6 \%$ e valor preditivo negativo de $98,5 \%$, e $89,9 \%$ de precisão no diagnóstico do TAS (Connor, Kobak, Churchill, Katzelnick, \& Davidson, 2001).

Inventário de Habilidades Sociais (IHS-Del-Prette). Instrumento de autorrelato para a avaliação das dimensões situacional e comportamental molar das habilidades sociais, contendo 38 itens, cada um deles descrevendo uma relação interpessoal e uma possível reação àquela situação. A resposta a cada item pode ser classificada de 0 (nunca ou raramente) até 4 (sempre ou quase sempre), em uma escala de 5 pontos, com escore total variando de 0 a 152. A análise das propriedades psicométricas, no estudo original, junto a 527 universitários obteve os seguintes resultados: índices de discriminação positivos, variando de 3,0 a 16,7; consistência interna satisfatória com um alfa de Cronbach de 0,75 ; e estrutura fatorial com cinco fatores principais, identificados em termos comportamentais-situacionais, com coeficientes alfa variando de 0,74 a 0,96 , que explicam $92,75 \%$ da variância total ( $\mathrm{Z}$. A. P. Del Prette et al., 1998). O IHS-Del-Prette apresenta a seguinte estrutura fatorial: (a) Fator 1 - Enfrentamento e auto-afirmação com risco (composto pelos itens 1, 5, 7, 11, 12, 14, 15, 16, 20, 21 e 29); (b) Fator 2 - Autoafirmação na expressão de sentimento positivo (itens 3 , 6, 8, 10, 28, 30 e 35); (c) Fator 3 - Conversação e desenvoltura social (itens 13, 17, 19, 22, 24, 36 e 37); (d) Fator 4 - Auto-exposição a desco-nhecidos e situações novas (itens 9, 14, 23 e 26); (e) Fator 5 - Autocontrole da agressividade (itens 18,31 e 38 ).
Entrevista Clínica Estruturada para o DSM-IV ([SCIDCV - versão clínica], First, Spitzer, Gibon, \& Willians, 1997), traduzida e adaptada para o português do Brasil (Del-Ben et al., 2001). Caracteriza-se como um instrumento utilizado para a elaboração de diagnósticos clínicos psiquiátricos baseados no DSM-IV. É composto por módulos, em um total de dez, que podem ser aplicados de forma independente ou combinada, conforme os objetivos almejados. Neste estudo foi utilizado o Módulo de Ansiedade (Módulo F), visando a confirmação diagnóstica do TAS nos sujeitos identificados com tal transtorno psiquiátrico (grupo caso) e nos sujeitos que constituíram o grupo de comparação (não-caso).

\section{Procedimento}

Em uma primeira instância, todos os instrumentos foram aplicados em situação coletiva de sala de aula, evitando-se dias de provas e sendo priorizadas as disciplinas com o maior número de alunos matriculados, após a assinatura do Termo de Consentimento Livre e Esclarecido. Contou-se sempre com a presença de dois aplicadores de modo a garantir a pronta assistência aos sujeitos quando solicitado.

Posteriormente, os nomes dos sujeitos foram incluídos em uma lista de números aleatórios e foram selecionados os 100 primeiros sujeitos a partir de seus escores na MiniSPIN, sendo 57 sujeitos com Mini-SPIN positiva (mínimo de seis pontos) e 43 com Mini-SPIN negativa (zero a um ponto). Estes sujeitos foram avaliados pela Entrevista Clínica Estruturada - Módulo F (SCID-IV), durante uma entrevista telefônica, objetivando-se a confirmação diagnóstica do TAS. Deste procedimento resultou uma amostra de 86 sujeitos, agrupados como caso $(n=45) \mathrm{e}$ não-caso de TAS $(n=41)$, e que contavam com os dois instrumentos, objeto deste estudo, preenchidos corretamente. No estudo de Crippa et al. (2008), entrevistas realizadas com 100 sujeitos, primeiramente por telefone e depois pessoalmente, demonstraram uma concordância excelente, com um coeficiente kappa de 0,84.

As duas amostras resultantes dos procedimentos de coleta de dados acima descritos (uma com 1006 e a outra com 86 sujeitos) foram independentemente estudadas a fim de verificar propriedades psicométricas do IHS-Del-Prette. A amostra de 100 sujeitos foi utilizada para verificar a sua validade concorrente e a amostra de 86 sujeitos, a validade discriminativa e preditiva do instrumento, bem como o poder discriminativo de cada um de seus itens.

\section{Tratamento de Dados}

Análise estatística descritiva (médias, desvios-padrões e porcentagens) e testes estatísticos paramétricos foram utilizados para o tratamento dos dados coletados. O nível de significância adotado nos testes estatísticos foi de $p \leq 0,05$. As análises efetuadas serão descritas a seguir. 
No que diz respeito à validade concorrente entre os instrumentos empregados, os escores do IHS-Del-Prette foram comparados com os escores do SPIN e Mini-SPIN, utilizando-se o coeficiente de Correlação Momento-Produto de Pearson. O coeficiente de correlação entre o IHSDel-Prette e a SPIN foi elevado ao quadrado para verificar quanta variância, em termos percentuais, as duas variáveis compartilham. Para determinar se a correlação da validade concorrente entre o IHS-Del-Prette e SPIN era significativamente maior do que a correlação da validade entre o IHS-Del-Prette e Mini-SPIN, foi aplicada a fórmula recomendada por J. Cohen e Cohen (1975) para testar a significância da diferença entre as duas correlações encontradas dentro da mesma amostra.

Para o estudo da validade discriminativa do IHS-DelPrette ou validade do instrumento para grupos conhecidos, foi obtida a curva ROC (Receiver Operating Characteristic Curve), visando examinar os pontos de corte que correspondem ao diagnóstico do TAS, distinguindo assim sujeitos com TAS daqueles sem o transtorno, tomando como padrão-ouro a Entrevista Clínica Estruturada (SCID-IV/DSM-IV). Foram determinados também os escores de corte que: (a) maximizam conjuntamente a sensibilidade, a especificidade, valor preditivo positivo (VPP), valor preditivo negativo (VPN), minimizando a taxa de classificação incorreta do IHS-Del-Prette; e (b) maximizam a sua sensibilidade sem, entretanto, reduzir a especificidade, VPP e VPN a níveis baixos.

Visando também à avaliação da validade discriminativa do IHS-Del-Prette, os escores totais do IHS-Del-Prette para os sujeitos agrupados como casos e não-casos de TAS foram comparados por meio do Teste $t$ de Student. Os sujeitos agrupados como casos e não-casos foram submetidos à confirmação diagnóstica de TAS por meio da SCID. Este mesmo teste foi aplicado sobre os escores médios de cada um dos itens obtidos para ambos os grupos, objetivando verificar se demonstrariam diferenças significativas nos itens isolados.

Visando identificar os itens do IHS-Del-Prette com maior poder discriminativo entre os sujeitos com e sem TAS $(N=86)$, foram avaliados quais itens apresentavam diferenças maiores nos escores médios entre os grupos (caso e não-caso). Observou-se também a porcentagem de pontuação para cada item nos escores menores ou iguais a 1 .

\section{Resultados}

\section{Validade Concorrente}

Uma boa validade concorrente foi demonstrada entre o IHS-Del-Prette e o SPIN, aplicados em 1006 sujeitos, com um coeficiente altamente significativo de correlação negativa entre estes dois instrumentos $(r=-0,68$, $p<0,001$ ), obtido pela comparação de seus escores totais, indicando que quanto mais elaborado for o repertório de habilidades sociais de um indivíduo, menor é a probabilidade de ele satisfazer os critérios de rastrea- mento de indicadores diagnósticos para o TAS.

Por conseguinte, verificou-se que $46 \%$ correspondem à variância que o IHS-Del-Prette e SPIN compartilham. Em outras palavras, que $46 \%$ da variabilidade nos indicadores detectados com a aplicação do SPIN, como característicos do quadro de TAS, para um dado indivíduo pode ser explicado pela variabilidade no seu repertório de habilidades sociais avaliado pelo IHS-Del-Prette. Sobram ainda 54\% que não é explicado, ou seja, a variância nos escores deve-se a outros fatores não explicados pelos instrumentos. Pode-se considerar assim que $27 \%$ da variância é exclusiva do IHS-Del-Prette e 27\% exclusiva do SPIN.

A validade concorrente foi testada ainda entre o IHSDel-Prette e o Mini-SPIN, com um forte relacionamento negativo obtido entre os dois $(r=-0,64, p<0,001)$, sugerindo que este instrumento em sua versão abreviada continua a produzir resultados significativos. Para confirmar este dado, o cálculo de significância da diferença entre os coeficientes de correlação demonstrou que a correlação entre o IHS-Del-Prette e o SPIN foi significativamente igual à correlação entre o IHS-Del-Prette e Mini-SPIN $\left(t_{(2010)}=5,28, p>0,99\right)$.

\section{Validade Discriminativa e Preditiva do IHS-Del-Prette}

Para a avaliação da validade discriminativa do IHSDel-Prette, foram utilizados os dados da amostra de 86 sujeitos submetidos à confirmação diagnóstica do TAS pela Entrevista Clínica Estruturada (SCID/DSM-IV). Inicialmente, realizou-se a análise dos dados por meio da curva ROC que se encontra ilustrada na Figura 1.

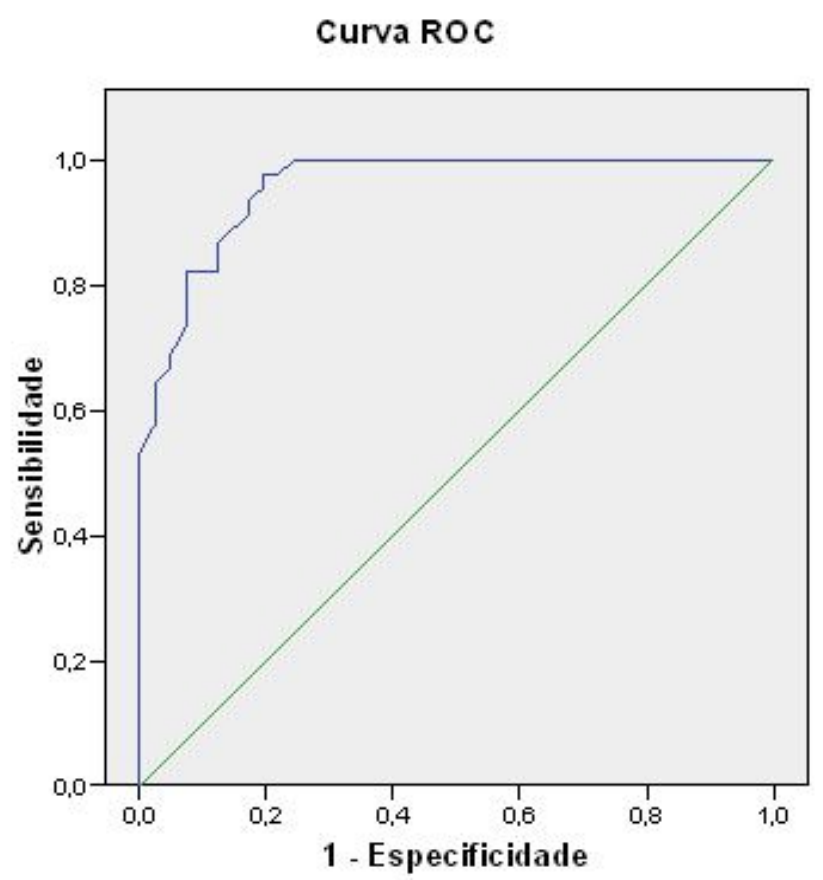

Figura 1. Area sob a curva ROC do escore total do IHS-DelPrette para uma amostra de casos e não-casos de TAS $(N=86)$. 
Angélico, A. P., Crippa, J. A. S. \& Loureiro, S. R. (2012). Utilização do Inventário de Habilidades Sociais no Diagnóstico do Transtorno de Ansiedade Social.

A área sob a curva encontrada foi de $0,96(p<0,001)$, com erro padrão de 0,02 , e intervalo de confiança de $95 \%$ entre 0,92 e 0,99. Calculou-se, também, a sensibilidade, especificidade, valor preditivo positivo, valor preditivo negativo e taxa de classificação incorreta para vários pontos (escores) de corte do IHS-Del-Prette, visando identificar aquele mais apropriado para o diagnóstico do TAS. Os dados obtidos são apresentados na Tabela 1.

Tabela 1

Valores Relativos à Sensibilidade, Especificidade, Valor Preditivo Positivo, Valor Preditivo Negativo e Taxa de Classificação Incorreta para Diferentes Pontos de Corte do IHS-Del-Prette em uma Amostra de Casos $(n=45)$ e nãocasos de TAS $(n=41)$

\begin{tabular}{cccccc}
\hline Escore de corte & Sensibilidade & Especificidade & VPP & VPN & TCI \\
\hline 80 & 0,73 & 0,93 & 0,92 & 0,76 & 0,17 \\
82 & 0,78 & 0,93 & 0,92 & 0,79 & 0,15 \\
83 & 0,82 & 0,93 & 0,92 & 0,83 & 0,13 \\
84 & 0,82 & 0,88 & 0,88 & 0,82 & 0,15 \\
85 & 0,84 & 0,88 & 0,88 & 0,84 & 0,14 \\
86 & 0,87 & 0,88 & 0,89 & 0,86 & 0,13 \\
87 & 0,89 & 0,85 & 0,87 & 0,88 & 0,13 \\
88 & 0,91 & 0,83 & 0,85 & 0,89 & 0,13 \\
89 & 0,93 & 0,83 & 0,86 & 0,92 & 0,12 \\
92 & 0,96 & 0,80 & 0,84 & 0,94 & 0,12 \\
94 & 0,98 & 0,80 & 0,85 & 0,97 & 0,10 \\
95 & 0,98 & 0,78 & 0,83 & 0,97 & 0,12 \\
97 & 1,00 & 0,76 & 0,82 & 1,00 & 0,12 \\
99 & 1,00 & 0,71 & 0,79 & 1,00 & 0,14 \\
102 & 1,00 & 0,63 & 0,75 & 1,00 & 0,17 \\
105 & 1,00 & 0,61 & 0,74 & 1,00 & 0,19 \\
\hline
\end{tabular}

Nota $. \mathrm{VPP}=$ valor preditivo positivo; VPN = valor preditivo negativo; TCI $=$ taxa de classificação incorreta.

Os escores de corte entre 88 e 95 foram aqueles que melhor equilibraram os valores de sensibilidade, especificidade, valor preditivo positivo e negativo, com taxa de classificação incorreta variando de 0,10 a 0,13 . Contudo, os valores entre os escores de corte 84 e 97 evidenciaram pouca variação e taxa de classificação incorreta variando de 0,10 a 0,15 . Em geral, quanto mais alto o valor critério selecionado (escore de corte), maior foi a sua sensibilidade e valor preditivo negativo e menor a especificidade e valor preditivo positivo. Para finalidades diagnósticas, o escore de corte 94 foi o que melhor distinguiu os indivíduos com e sem TAS, maximizando os valores de sua sensibilidade e especificidade e com uma taxa de classificação incorreta menor.

Para o escore de corte 102, observou-se uma diminuição em torno de $17 \%$ na especificidade e $10 \%$ no valor preditivo positivo, mantendo-se a sensibilidade e o valor preditivo negativo bastante expressivos, com uma taxa de classificação incorreta de 0,17 . Já o escore 82 favoreceu um aumento em torno de $13 \%$ para a especificidade e 7\% para o valor preditivo positivo, e uma diminuição em torno de $18 \%$ para a sensibilidade e $15 \%$ para o valor preditivo negativo, com uma taxa de classificação incorreta de 0,15 .
O IHS-Del-Prette demonstrou distinguir significativamente indivíduos com e sem TAS $\left(t_{(84)}=11,61, p<0,001\right)$, avaliados pela SCID, atestando assim sua validade discriminativa e preditiva para esse diagnóstico. A diferença entre as médias dos dois grupos foi de 39,96, que é um grande efeito $(d=16,41)$, segundo a classificação de J. Cohen (1988).

Ainda com relação à validade discriminativa do IHSDel-Prette, o grupo caso $(n=45)$ teve uma média de 67,84 $(D P=15,83)$ para os escores totais comparada com 107,80 $(D P=16,07)$ do grupo não-caso $(n=41)$. Em contrapartida, o grupo caso obteve uma média de $36,58(D P=$ $11,75)$ para os escores totais do SPIN comparada com $7,80(D P=4,29)$ do grupo não-caso.

Os escores médios de cada um dos itens do IHS-DelPrette, com seus respectivos desvios-padrões, valores $t \mathrm{e}$ probabilidades associadas foram calculados para os grupos caso $(n=45)$ e não-caso $(n=41)$, conforme apresentado na Tabela 2. 
Tabela 2

Escores Médios de cada Item do IHS-Del-Prette com seus Respectivos Desvios-Padrões e Testes t para os Grupos Caso $(n=45)$ e Não-Caso de TAS $(n=41)$

\begin{tabular}{|c|c|c|c|c|}
\hline Itens do IHS-Del-Prette & Grupo Caso & Grupo Não-Caso & Teste $t\left(t_{(84)}=\right)$ & Probabilidade \\
\hline 1. Manter conversa com desconhecidos & $0,78(0,93)$ & $2,32(1,19)$ & 6,64 & $p<0,001^{(\mathrm{S})}$ \\
\hline 2. Pedir mudança de conduta & $2,42(1,29)$ & $2,76(0,94)$ & 1,38 & $p=0,171^{(\mathrm{NS})}$ \\
\hline 3. Agradecer elogios & $3,09(1,08)$ & $3,29(1,08)$ & 0,87 & $p=0,385^{(\mathrm{NS})}$ \\
\hline 4. Interromper a fala do outro & $0,89(1,21)$ & $1,88(1,19)$ & 3,82 & $p<0,001^{(\mathrm{S})}$ \\
\hline 5. Cobrar dívida de amigo & $1,04(1,15)$ & $2,05(1,28)$ & 3,83 & $p<0,001^{(\mathrm{S})}$ \\
\hline 6. Elogiar outrem & $2,31(1,29)$ & $2,95(1,02)$ & 2,56 & $p=0,012^{(\mathrm{S})}$ \\
\hline 7. Apresentar-se a outra pessoa & $0,56(1,08)$ & $1,54(1,19)$ & 4,02 & $p<0,001^{(\mathrm{S})}$ \\
\hline 8. Participar de conversação & $2,04(1,31)$ & $3,49(0,95)$ & 5,87 & $p<0,001^{(\mathrm{S})}$ \\
\hline 9. Falar a público desconhecido & $1,11(1,25)$ & $2,98(1,06)$ & 7,43 & $p<0,001^{(\mathrm{S})}$ \\
\hline 10. Expressar sentimento positivo & $1,64(1,33)$ & $2,51(1,25)$ & 3,11 & $p=0,003^{(\mathrm{S})}$ \\
\hline 11. Discordar de autoridade & $0,78(1,06)$ & $2,20(1,40)$ & 5,25 & $p<0,001^{(\mathrm{S})}$ \\
\hline 12. Abordar para relação sexual & $0,51(0,92)$ & $1,71(1,35)$ & 4,76 & $p<0,001^{(\mathrm{S})}$ \\
\hline 13. Reagir a elogio & $2,00(1,19)$ & $3,12(1,12)$ & 4,49 & $p<0,001^{(\mathrm{S})}$ \\
\hline 14. Falar a público conhecido & $1,42(1,34)$ & $2,90(1,20)$ & 5,38 & $p<0,001^{(\mathrm{S})}$ \\
\hline 15. Lidar com críticas injustas & $2,33(1,40)$ & $3,17(1,24)$ & 2,94 & $p=0,004^{(\mathrm{S})}$ \\
\hline 16. Discordar do grupo & $1,93(1,23)$ & $3,20(1,08)$ & 5,03 & $p<0,001^{(\mathrm{S})}$ \\
\hline 17. Encerrar conversação & $1,91(1,10)$ & $3,27(0,90)$ & 6,22 & $p<0,001^{(\mathrm{S})}$ \\
\hline 18. Lidar com crítica dos pais & $2,82(1,05)$ & $3,27(0,67)$ & 2,37 & $p=0,021^{(\mathrm{S})}$ \\
\hline 19. Abordar autoridade & $1,04(1,30)$ & $2,98(1,01)$ & 7,65 & $p<0,001^{(\mathrm{S})}$ \\
\hline 20. Declarar sentimento amoroso & $1,29(1,31)$ & $2,59(1,20)$ & 4,77 & $p<0,001^{(\mathrm{S})}$ \\
\hline 21. Devolver mercadoria defeituosa & $2,04(1,31)$ & $3,39(0,95)$ & 5,49 & $p<0,001^{(\mathrm{S})}$ \\
\hline 22. Recusar pedidos abusivos & $1,60(1,37)$ & $2,20(1,23)$ & 2,11 & $p=0,038^{(\mathrm{S})}$ \\
\hline 23. Fazer pergunta a desconhecido & $1,64(1,25)$ & $3,20(0,87)$ & 6,73 & $p<0,001^{(\mathrm{S})}$ \\
\hline 24. Encerrar conversa ao telefone & $2,04(1,41)$ & $3,39(0,89)$ & 5,33 & $p<0,001^{(\mathrm{S})}$ \\
\hline 25. Lidar com críticas justas & $2,27(1,16)$ & $3,02(0,88)$ & 3,44 & $p=0,001^{(\mathrm{S})}$ \\
\hline 26. Pedir favores a desconhecidos & $1,91(1,44)$ & $3,00(1,12)$ & 3,93 & $p<0,001^{(\mathrm{S})}$ \\
\hline 27. Expressar desagrado a amigos & $1,24(1,32)$ & $2,07(1,21)$ & 3,03 & $p=0,003^{(\mathrm{S})}$ \\
\hline 28. Elogiar familiares & $2,91(0,95)$ & $3,34(0,66)$ & 2,42 & $p=0,018^{(\mathrm{S})}$ \\
\hline 29. Fazer pergunta a conhecidos & $1,22(1,06)$ & $2,80(1,19)$ & 6,52 & $p<0,001^{(\mathrm{S})}$ \\
\hline 30. Defender outrem em grupo & $1,69(1,18)$ & $2,71(1,23)$ & 3,91 & $p<0,001^{(\mathrm{S})}$ \\
\hline 31. Cumprimentar desconhecidos & $1,44(1,14)$ & $2,46(1,23)$ & 3,99 & $p<0,001^{(\mathrm{S})}$ \\
\hline 32. Pedir ajuda a amigos & $1,56(1,29)$ & $2,93(1,33)$ & 4,85 & $p<0,001^{(\mathrm{S})}$ \\
\hline 33. Negociar uso de preservativo & $3,53(0,92)$ & $3,63(0,77)$ & 0,55 & $p=0,584^{(\mathrm{NS})}$ \\
\hline 34. Recusar pedido abusivo & $2,13(1,42)$ & $3,07(1,06)$ & 3,49 & $p=0,001^{(\mathrm{S})}$ \\
\hline 35. Expressar sentimento positivo & $2,38(1,28)$ & $3,17(1,02)$ & 3,18 & $p=0,002^{(\mathrm{S})}$ \\
\hline 36. Manter conversação & $1,71(1,20)$ & $2,95(0,97)$ & 5,28 & $p<0,001^{(\mathrm{S})}$ \\
\hline 37. Pedir favores a colegas & $2,29(1,08)$ & $3,39(0,95)$ & 5,01 & $p<0,001^{(\mathrm{S})}$ \\
\hline 38. Lidar com chacotas & $2,29(1,29)$ & $2,93(1,06)$ & 2,52 & $p=0,014^{(\mathrm{S})}$ \\
\hline
\end{tabular}

Nota $. \mathrm{S}=$ diferença significativa; $\mathrm{NS}=$ diferença não significativa.

Observou-se diferença significativa entre os grupos em 35 das habilidades sociais avaliadas. Para o grupo caso, as habilidades sociais com escores mais altos foram as representadas pelos itens 33, 3, 28 e 18 . Considerando o grupo não-caso, as habilidades sociais com escores mais altos foram aquelas representadas pelos itens $33,8,37$, $24,21,28,3,18$ e 17. Todas as habilidades com escores mais altos apresentadas pelo grupo caso foram compartilhadas pelo grupo não-caso. Apenas em relação aos itens
2, 3 e 33, os dois grupos não diferiram, demonstrando assim uma diferença significativa para a maioria das habilidades sociais, com pontuações médias consideravelmente mais altas para o grupo não-caso em todas as habilidades.

No que diz respeito ao poder discriminativo dos itens, o escore médio e a porcentagem de freqüência nos escores de 0 a 4 em cada item, para os grupos caso e não-caso de TAS, são exibidos na Tabela 3 . 
Angélico, A. P., Crippa, J. A. S. \& Loureiro, S. R. (2012). Utilização do Inventário de Habilidades Sociais no Diagnóstico do Transtorno de Ansiedade Social.

Tabela 3

Distribuição dos Itens do IHS-Del-Prette em Função do Escore Médio, Desvio-Padrão e Porcentagem de Freqüência nos Escores de 0 a 4 para os Grupos Caso $(n=45)$ e Não-Caso de TAS $(n=41)$

\begin{tabular}{|c|c|c|c|c|c|c|c|c|c|c|c|c|c|c|}
\hline \multirow{3}{*}{$\begin{array}{l}\text { Item IHS- } \\
\text { Del-Prette }\end{array}$} & \multicolumn{7}{|c|}{ GRUPO CASO } & \multicolumn{6}{|c|}{ GRUPO NÃO-CASO } & \\
\hline & \multirow{2}{*}{1} & \multirow{2}{*}{$D P$} & \multicolumn{5}{|c|}{ Escores $(\%)$} & \multirow{2}{*}{$M$} & \multirow{2}{*}{$D P$} & \multicolumn{5}{|c|}{ Escores $(\%)$} \\
\hline & & & 0 & 1 & 2 & 3 & 4 & & & 0 & 1 & 2 & 3 & 4 \\
\hline 1 & 0,78 & 0,93 & 46,7 & 35,6 & 13,3 & 2,2 & 2,2 & 2,32 & 1,19 & 2,4 & 31,7 & 17,1 & 29,3 & 19,5 \\
\hline 2 & 2,42 & 1,29 & 11,1 & 11,1 & 26,7 & 26,7 & 24,4 & 2,76 & 0,94 & 4,9 & 2,4 & 21,9 & 53,7 & 17,1 \\
\hline 3 & 3,09 & 1,08 & 2,2 & 8,9 & 13,3 & 28,9 & 46,7 & 3,29 & 1,08 & 0,0 & 12,2 & 9,8 & 14,6 & 63,4 \\
\hline 4 & 0,89 & 1,21 & 48,9 & 33,3 & 6,7 & 2,2 & 8,9 & 1,88 & 1,19 & 9,8 & 34,1 & 26,8 & 17,1 & 12,2 \\
\hline 5 & 1,04 & 1,15 & 42,2 & 28,9 & 13,4 & 13,3 & 2,2 & 2,05 & 1,28 & 12,2 & 24,4 & 26,8 & 19,5 & 17,1 \\
\hline 6 & 2,31 & 1,29 & 11,1 & 15,6 & 26,7 & 24,4 & 22,2 & 2,95 & 1,02 & 2,4 & 7,3 & 17,1 & 39,0 & 34,2 \\
\hline 7 & 0,56 & 1,08 & 68,9 & 20,0 & 4,4 & 0,0 & 6,7 & 1,54 & 1,19 & 22,0 & 31,6 & 22,0 & 19,5 & 4,9 \\
\hline 8 & 2,04 & 1,31 & 17,8 & 13,3 & 31,1 & 22,2 & 15,6 & 3,49 & 0,95 & 4,9 & 0,0 & 2,4 & 26,8 & 65,9 \\
\hline 9 & 1,11 & 1,25 & 40,0 & 31,1 & 15,6 & 4,4 & 8,9 & 2,98 & 1,06 & 4,9 & 2,4 & 19,5 & 36,6 & 36,6 \\
\hline 10 & 1,64 & 1,33 & 24,4 & 26,7 & 20,0 & 17,8 & 11,1 & 2,51 & 1,25 & 4,9 & 21,9 & 17,1 & 29,3 & 26,8 \\
\hline 11 & 0,78 & 1,06 & 55,6 & 22,2 & 13,3 & 6,7 & 2,2 & 2,20 & 1,40 & 12,2 & 26,8 & 14,6 & 22,0 & 24,4 \\
\hline 12 & 0,51 & 0,92 & 68,9 & 17,8 & 8,9 & 2,2 & 2,2 & 1,71 & 1,35 & 24,4 & 24,4 & 17,1 & 24,4 & 9,7 \\
\hline 13 & 2,00 & 1,19 & 11,1 & 26,7 & 22,2 & 31,1 & 8,9 & 3,12 & 1,12 & 2,4 & 9,8 & 12,2 & 24,4 & 51,2 \\
\hline 14 & 1,42 & 1,34 & 26,7 & 40,0 & 11,1 & 8,9 & 13,3 & 2,90 & 1,20 & 2,4 & 14,6 & 17,1 & 22,0 & 43,9 \\
\hline 15 & 2,33 & 1,40 & 11,1 & 24,4 & 11,1 & 26,7 & 26,7 & 3,17 & 1,24 & 7,3 & 7,3 & 2,5 & 26,8 & 56,1 \\
\hline 16 & 1,93 & 1,23 & 15,6 & 20,0 & 31,1 & 22,2 & 11,1 & 3,20 & 1,08 & 2,4 & 7,3 & 12,2 & 24,4 & 53,7 \\
\hline 17 & 1,91 & 1,10 & 11,1 & 24,4 & 33,4 & 24,4 & 6,7 & 3,27 & 0,90 & 2,4 & 2,4 & 7,4 & 41,5 & 46,3 \\
\hline 18 & 2,82 & 1,05 & 2,2 & 11,1 & 17,8 & 40,0 & 28,9 & 3,27 & 0,67 & 0,0 & 0,0 & 12,2 & 48,8 & 39,0 \\
\hline 19 & 1,04 & 1,30 & 46,7 & 26,7 & 11,0 & 6,7 & 8,9 & 2,98 & 1,01 & 0,0 & 14,6 & 7,3 & 43,9 & 34,2 \\
\hline 20 & 1,29 & 1,31 & 35,6 & 28,9 & 15,6 & 11,0 & 8,9 & 2,59 & 1,20 & 0,0 & 26,8 & 19,5 & 22,0 & 31,7 \\
\hline 21 & 2,04 & 1,31 & 11,1 & 31,1 & 17,8 & 22,2 & 17,8 & 3,39 & 0,95 & 2,4 & 2,4 & 9,8 & 24,4 & 61,0 \\
\hline 22 & 1,60 & 1,37 & 31,1 & 15,6 & 26,6 & 15,6 & 11,1 & 2,20 & 1,23 & 12,2 & 14,6 & 29,3 & 29,3 & 14,6 \\
\hline 23 & 1,64 & 1,25 & 22,2 & 24,4 & 28,9 & 15,6 & 8,9 & 3,20 & 0,87 & 0,0 & 4,9 & 14,6 & 36,6 & 43,9 \\
\hline 24 & 2,04 & 1,41 & 20,0 & 15,6 & 24,4 & 20,0 & 20,0 & 3,39 & 0,89 & 2,4 & 2,4 & 4,9 & 34,2 & 56,1 \\
\hline 25 & 2,27 & 1,16 & 6,6 & 20,0 & 28,9 & 28,9 & 15,6 & 3,02 & 0,88 & 0,0 & 7,3 & 14,6 & 46,4 & 31,7 \\
\hline 26 & 1,91 & 1,44 & 22,2 & 22,2 & 15,6 & 22,2 & 17,8 & 3,00 & 1,12 & 4,9 & 2,4 & 24,4 & 24,4 & 43,9 \\
\hline 27 & 1,24 & 1,32 & 35,6 & 33,3 & 13,3 & 6,7 & 11,1 & 2,07 & 1,21 & 4,9 & 36,6 & 22,0 & 19,5 & 17,0 \\
\hline 28 & 2,91 & 0,95 & 0,0 & 8,9 & 22,2 & 37,8 & 31,1 & 3,34 & 0,66 & 0,0 & 0,0 & 9,8 & 46,3 & 43,9 \\
\hline 29 & 1,22 & 1,06 & 28,9 & 33,4 & 28,9 & 4,4 & 4,4 & 2,80 & 1,19 & 0,0 & 17,1 & 29,3 & 9,8 & 43,8 \\
\hline 30 & 1,69 & 1,18 & 20,0 & 24,4 & 26,8 & 24,4 & 4,4 & 2,71 & 1,23 & 4,9 & 14,6 & 19,5 & 26,8 & 34,2 \\
\hline 31 & 1,44 & 1,14 & 26,7 & 24,4 & 28,9 & 17,8 & 2,2 & 2,46 & 1,23 & 2,5 & 26,8 & 19,5 & 24,4 & 26,8 \\
\hline 32 & 1,56 & 1,29 & 22,2 & 33,3 & 24,5 & 6,7 & 13,3 & 2,93 & 1,33 & 7,3 & 12,1 & 9,8 & 22,0 & 48,8 \\
\hline 33 & 3,53 & 0,92 & 2,2 & 2,2 & 8,9 & 13,4 & 73,3 & 3,63 & 0,77 & 0,0 & 4,9 & 2,4 & 17,1 & 75,6 \\
\hline 34 & 2,13 & 1,42 & 17,8 & 17,8 & 20,0 & 22,2 & 22,2 & 3,07 & 1,06 & 2,5 & 7,3 & 14,6 & 31,7 & 43,9 \\
\hline 35 & 2,38 & 1,28 & 8,8 & 15,6 & 31,1 & 17,8 & 26,7 & 3,17 & 1,02 & 2,4 & 4,9 & 14,6 & 29,3 & 48,8 \\
\hline 36 & 1,71 & 1,20 & 17,8 & 28,9 & 24,4 & 22,2 & 6,7 & 2,95 & 0,97 & 2,4 & 7,3 & 12,2 & 48,8 & 29,3 \\
\hline 37 & 2,29 & 1,08 & 4,5 & 20,0 & 31,1 & 31,1 & 13,3 & 3,39 & 0,95 & 0,0 & 7,3 & 9,8 & 19,5 & 63,4 \\
\hline 38 & 2,29 & 1,29 & 11,1 & 17,8 & 22,2 & 28,9 & 20,0 & 2,93 & 1,06 & 4,9 & 4,9 & 14,6 & 43,9 & 31,7 \\
\hline
\end{tabular}

Nota. $D P=$ desvio-padrão; $\%=$ Porcentagem.

Os itens $1,8,9,11,14,19,23$ e 29 foram os oito primeiros com maior diferença entre as médias de pontuação nos escores $(1,54,1,45,1,87,1,42,1,48,1,94,1,56 \mathrm{e}$ 1,58 , respectivamente). Para o grupo caso, esses itens apresentaram os maiores percentuais de pontuação nos escores menores ou iguais a um em comparação ao grupo não-caso, a saber: $82,3,31,1,71,1,77,8,66,7,73,4$, 46,6 e $62,3 \%$, respectivamente. Assim sendo, os itens 19 ,
$9,29,23,1,14,8$ e 11 foram, ordenadamente, os oito itens que evidenciaram maior poder discriminativo para os grupos caso e não-caso de TAS. Não obstante, para o grupo não-caso, os itens $8,9,19$ e 23 apresentaram percentuais bastante expressivos nos escores maiores ou iguais a três: $92,7,73,2,78,1$ e $80,5 \%$, respectivamente, corroborando, deste modo, o poder discriminativo destes itens. 
Os itens 20,21, 24, 17 e 32 também apresentaram poder discriminativo relativamente alto, com uma diferença entre as médias de pontuação nos escores registrados pelos dois grupos variando de 1,30 a 1,37. Estes itens receberam os seguintes percentuais de pontuação nos escores menores ou iguais a um: 64,5, 42,2, 35,6, 35,5 e $55,5 \%$, respectivamente. Para o grupo não-caso, os itens $21,24,17$ e 32 demonstraram percentuais bastante expressivos nos escores maiores ou iguais a três: 85,4, 90,3, 87,8 e $70,8 \%$, respectivamente, confirmando assim o poder discriminativo destes itens.

\section{Discussão}

A boa validade concorrente demonstrada entre o IHSDel-Prette e o SPIN evidenciou que quanto mais elaborado for o repertório de habilidades sociais de um indivíduo, menor será a probabilidade de ele satisfazer os critérios de rastreamento de indicadores diagnósticos para o TAS. Neste sentido, a hipótese inicial do estudo foi confirmada, ou seja, as manifestações comportamentais identificadas com a aplicação do IHS-Del-Prette estão de fato associadas às manifestações clínicas detectadas com a aplicação do SPIN. Deste modo, pode-se dizer que os resultados obtidos apontam para a relação existente entre habilidades sociais, funcionamento social e sintomas do TAS. Os 54\% da variância exclusiva do IHS-Del-Prette e SPIN ( $27 \%$ de um e $27 \%$ do outro), que não puderam ser explicados pela variância compartilhada pelos instrumentos, devem-se a outros fatores que podem abranger os eventos ambientais tanto mais imediatos na vida de um indivíduo quanto relacionados à sua história de desenvolvimento socioemocional, ou ainda uma conjunção de ambos.

Uma observação precisa ser feita quanto à forte relação negativa também encontrada entre o IHS-Del-Prette e a Mini-SPIN, sugerindo que esta escala em sua versão abreviada continua a produzir resultados significativos. Não obstante, para efeitos de uma melhor avaliação dos indicadores diagnósticos do TAS, ainda que na condição de rastreamento, o SPIN pode fornecer uma caracterização mais completa e específica das manifestações do TAS apresentadas, visto que avalia um número maior de eventos e situações eliciadoras dos sinais e sintomas apresentados por um indivíduo.

No tocante à validade discriminativa do IHS-Del-Prette, os resultados mostraram que os indivíduos com TAS diferiram significativamente de outros indivíduos sem TAS ou qualquer outro transtorno psiquiátrico em termos de habilidades sociais. Em acréscimo, como esperado, os indivíduos com TAS obtiveram escores mais baixos no IHSDel-Prette e mais altos no SPIN quando comparados com indivíduos sem este diagnóstico, corroborando assim a correlação inversa verificada entre os dois instrumentos.

A área sob a curva de 0,96 $(p<0,001)$ encontrada para os dados do IHS-Del-Prette indicou uma precisão alta deste instrumento para discriminar casos e não-casos de TAS. Deste modo, a área de 0,96 significa que um indiví- duo selecionado randomicamente do grupo caso tem um valor (escore) de teste menor do que aquele obtido por um indivíduo escolhido randomicamente do grupo nãocaso em $96 \%$ das vezes. Além disso, a análise da curva ROC indicou que o escore de corte 94 foi o que melhor distinguiu os indivíduos com e sem TAS, produzindo um melhor equilíbrio entre a sensibilidade e especificidade, com uma taxa de classificação incorreta menor. No entanto, o escore de corte 97 evidenciou ser o mais adequado para propósitos de rastreamento, ou seja, com uma sensibilidade e valor preditivo negativo mais alto.

Os itens 1 (manter conversa com desconhecidos) e 23 (fazer pergunta a desconhecido) evidenciaram maior poder discriminativo para os sujeitos dos grupos caso e não-caso de TAS, submetidos à confirmação diagnóstica, e tal dado está consistente com os resultados de Stopa e Clark (1993). Os resultados obtidos por esses autores demonstraram que, em uma situação social hipotética de encontrar dois velhos amigos de uma pessoa conhecida, quando na companhia dela em um bar, os fóbicos sociais tiveram mais pensamentos autoavaliativos negativos comparados aos controles ansiosos e não-pacientes. Neste caso, os fóbicos sociais também tiveram mais pensamentos acerca de evitação e poucos sobre planejamento (pensamentos sobre estratégias de enfrentamento e objetivos comportamentais) do que os controles. Esse foco em pensamentos negativos interfere com o comportamento adaptativo, impedindo o sujeito de formular um plano de como lidar adequadamente com o encontro e, assim, aumentando a ansiedade e estimulando mais julgamentos negativos sobre si mesmo.

A habilidade de negociar uso de preservativo, encontrada em comum para os grupos caso e não-caso, submetidos à SCID, assume relevância não só social como também profilática, considerando dois fatores: (a) ambos os grupos são provindos de uma amostra pertencente a uma faixa etária jovem que estimula desde contatos meramente sociais até práticas sexuais; e (b) compartilham uma parte significativa de suas vidas dentro do contexto universitário, um ambiente favorável também para novos contatos interpessoais e práticas sexuais. Com o avanço das doenças sexualmente transmissíveis e, particularmente, com a disseminação da AIDS, essa habilidade torna-se cada vez mais requerida tanto para a consolidação de uma relação como para a garantia da saúde. Conforme apontam A. Del Prette e Del Prette (2001), solicitar ou exigir o uso de preservativo e recusar sexo inseguro, dentre outras medidas, constituem habilidades de enfrentamento que precisam ser desenvolvidas e exercitadas. Embora os dois grupos não tenham demonstrado diferença para a habilidade de negociar o uso de preservativo, atestaram diferenças significativas para as habilidades de abordar para relação sexual e declarar sentimento amoroso que do mesmo modo estão inseridas no contexto do relacionamento afetivo/sexual.

Do rol de habilidades sociais que os dois grupos demonstraram diferenças significativas, observou-se que 
essas habilidades estão relacionadas a diferentes: (a) interlocutores, como parceiro sexual (itens 12 e 20), autoridades (11 e 19), familiar (10, 15, 18 e 28), desconhecidos $(1,7,9,21,23,26,31$ e 36), conhecidos $(8,13$, $14,16,22,24,29,34,37$ e 38), amigos $(5,17,27,32$ e 35 ) e indefinido (4, 6, 25 e 30); e (b) contextos, entre eles o público, como de trabalho, estudo ou consumo (itens 1 , $5,8,9,11,13,14,16,19,21,22,23,26,29,30,31,37$ e $38)$, o privado, como o doméstico ou íntimo $(2,10,12$, $15,17,18,20,28,34,35$, e 36) e indefinido $(3,4,6,7$, 24, 25, 27 e 32). Assim sendo, esses resultados evidenciam claramente a relação entre os possíveis déficits em habilidades sociais e prejuízos diversos no funcionamento social que indivíduos com TAS podem experimentar em seu dia-a-dia.

O perfil amostral deste estudo caracterizou-se exclusivamente por estudantes universitários do interior do estado de São Paulo e, embora com uma faixa etária bem ampla, este fato impossibilita a generalização dos resultados obtidos para outras amostras com diferentes níveis educacionais e advindas de outras regiões culturalmente distintas. Sendo assim, esse perfil amostral circunscrito torna relativa a validade externa deste estudo, ou seja, o grau em que os seus resultados podem ser generalizados para outras populações, situações ou contextos. Em outras palavras, a exemplo de inúmeros estudos conduzidos com amostras de conveniência em um único centro, e em conformidade com Selltiz, Wrightsman e Cook (1987), se os resultados fossem verdadeiros não apenas para o momento, lugar e pessoas do estudo, mas também o fossem para outros momentos, lugares e pessoas, o estudo poderia ter uma maior validade externa.

No entanto, este estudo atendeu algumas recomendações metodológicas para garantir a qualidade dos dados obtidos (Hansson, 2001; Perreault, Katerelos, Sabourin, Leichner, \& Desmarais, 2001; Ruggeri, 1994; Smith, Manderscheid, Flynn, \& Steinwatchs, 1997). O estudo utilizou instrumentos de medida padronizados e que têm suas qualidades psicométricas aferidas, em termos de sua validade e fidedignidade. Instrumentos de medida validados permitem a confiabilidade dos dados e a comparabilidade dos resultados em diferentes pesquisas. Além disso, o IHS-Del-Prette e o SPIN constituem instrumentos de avaliação bastante adequados, considerando que são escalas multidimensionais, validadas, que possuem vários itens e alternativas de respostas variadas para cada item, o que possibilita uma maior sensibilidade das medidas na detecção de diferentes níveis dos construtos avaliados.

Por outro lado, considera-se que uma avaliação clínica ampla seja imprescindível na decisão de qual abordagem de tratamento será a mais apropriada para um dado indivíduo com TAS. Uma avaliação completa incluiria uma entrevista estruturada ou semi-estruturada, medidas de autorrelato e avaliação comportamental. Cada uma destas medidas forneceria diferentes tipos de informação que podem ser relevantes para decisões posteriores quanto ao tratamento. Nesta direção, este estudo contou com a aplicação da SCID, SPIN e IHS-Del-Prette que, conforme o contexto, poderiam conjuntamente constituir medidas diretas, objetivas e confiáveis para uma avaliação completa e específica no diagnóstico do TAS e, conseqüentemente, para o planejamento de medidas de intervenção mais apropriadas às necessidades dos indivíduos com tal transtorno.

Em particular, o IHS-Del-Prette pode ser empregado como um forte aliado do SPIN, que avalia o espectro completo dos sintomas em termos de medo, evitação e componentes fisiológicos, para a obtenção de uma avaliação mais completa e específica dos indicadores diagnósticos apresentados pelos indivíduos com fobia social.

Estudos adicionais serão necessários para abordar aspectos não contemplados e limitações do estudo em questão. São estes:

1.A avaliação da validade discriminativa e preditiva do IHS-Del-Prette ganharia uma maior consistência e respaldo se realizada com uma amostra mais ampla de sujeitos diagnosticados como casos e não-casos de TAS, deixando esta questão em aberto para futuros estudos;

2.Faltou uma maior abrangência do estudo para determinar se a escala discriminaria participantes socialmente fóbicos daqueles com outros transtornos de ansiedade e/ou com outros quadros diagnósticos, como a depressão;

3.O IHS-Del-Prette foi validado, neste estudo, como uma escala para selecionar participantes socialmente fóbicos, mas ainda não está claro se esta escala é capaz de distinguir participantes com TAS do subtipo generalizado daqueles do subtipo circunscrito ou específico;

4.Verificou-se a necessidade de avaliar a sensibilidade do IHS-Del-Prette à redução de déficits interpessoais e sintomas apresentados pelos indivíduos diagnosticados com esse transtorno ao longo do tempo, principalmente após a aplicação de intervenções farmacológicas e/ou programas de tratamento, como por exemplo, o Treinamento em Habilidades Sociais. Neste caso, em particular, estar-se-ia testando a validade do instrumento para detectar as possíveis mudanças produzidas por tais abordagens de intervenção. No entanto, poder-se-ia testar também a validade do tratamento (ou terapêutica) avaliada pelo instrumento por meio da SCID, visando verificar, assim, a sua validade de dupla face nesse quesito.

\section{Conclusões}

Os resultados apontaram que o IHS-Del-Prette pode ser um instrumento apropriado para detectar as dificuldades ou déficits interpessoais que indivíduos com TAS apresentam, constituindo uma ferramenta útil para uma melhor caracterização do seu quadro diagnóstico, ou mais especificamente para a avaliação dos seus déficits de de- 
sempenho em habilidades sociais. Evidencia-se assim tanto a sua validade discriminativa e preditiva no diagnóstico deste transtorno, quanto a possibilidade de uso do instrumento em estudos empíricos que testem a eficácia terapêutica de programas de intervenção.

\section{Referências}

American Psychiatric Association. (1994). DSM-IV: Diagnostic and statistical manual of mental disorders $\left(4^{\text {th }}\right.$ ed. $)$. Washington, DC: Author.

Angélico, A. P., Crippa, J. A. S., \& Loureiro, S. R. (2006, jan./ jun.). Fobia social e habilidades sociais: Uma revisão da literatura. Interação em Psicologia (Curitiba), 10(1), 113-125.

Ayres, L. S. M. (1994). Uma escala brasileira para a medida da assertividade. (Dissertação de Mestrado não-publicada). Universidade Gama Filho, Rio de Janeiro, RJ.

Bandeira, M. (2002, nov.). Escala de Avaliação da Competência Social de Pacientes Psiquiátricos através de Desempenho de Papéis - EACS. Avaliação Psicológica, 1(2), 159-171.

Cohen, J. (1988). Statistical power for Behavioral Sciences $\left(2^{\text {nd }}\right.$ ed.). New York: Academic Press.

Cohen, J., \& Cohen, P. (1975). Applied multiple regression/ correlation analysis for the behavioral sciences. Hillsdale, NJ: Lawrence Erlbaum.

Connor, K. M., Davidson, J. R. T., Churchill, L. E., Sherwood, A., Foa, E., \& Weisler, R. H. (2000, April). Psychometric properties of the Social Phobia Inventory (SPIN): A new selfrating scale. British Journal of Psychiatry, 176, 379-386.

Connor, K. M., Kobak, K. A., Churchill, L. E., Katzelnick, D., \& Davidson, J. R. T. (2001). Mini-SPIN: A brief screening assessment for generalized social anxiety disorder. Depression and Anxiety, 14(2), 137-140.

Crippa, J. A. S., de Lima Osório, F., Del-Ben, C. M., Filho, A. S., da Silva Freitas, M. C., \& Loureiro, S. R. (2008). Comparability between telephone and face-to-face structured clinical interview for DSM-IV in assessing social anxiety disorder. Perspectives in Psychiatric Care, 44(4), 241-247.

Del Prette, A., \& Del Prette, Z. A. P. (2001). Psicologia das relações interpessoais: Vivências para o trabalho em grupo. Petrópolis, RJ: Vozes.

Del Prette, Z. A. P., \& Del Prette, A. (2001). Inventário de Habilidades Sociais (IHS-Del-Prette): Manual de aplicação, apuração e interpretação. São Paulo, SP: Casa do Psicólogo.

Del Prette, Z. A. P., \& Del Prette, A. (2005). Psicologia das habilidades sociais na infância: Teoria e prática. Petrópolis, RJ: Vozes.

Del Prette, Z. A. P., Del Prette, A., \& Barreto, M. C. M. (1998). Análise de um Inventário de Habilidades Sociais (IHS) em uma amostra de universitários. Psicologia: Teoria e Pesquisa, 14(3), 219-228.

Del-Ben, C. M., Vilela, J. A. A., Crippa, J. A. S., Hallak, J. E. C., Labate, C. M., \& Zuardi, A. W. (2001, set.). Confiabilidade da entrevista clínica estruturada para o DSM-IV - Versão clínica traduzida para o português. Revista Brasileira de Psiquiatria, 23(3), 156-159.

Falcone, E. M. O. (1998). Fobia social. In B. Rangé (Ed.), Psicoterapia comportamental e cognitiva de transtornos psiquiátricos (pp. 133-149). Campinas, SP: Psy.

Falcone, E., \& Figueira, I. (2001). Transtorno de ansiedade social. In B. Rangé (Ed.), Psicoterapias cognitivo-comportamentais: Um diálogo com a psiquiatria (pp. 183-207). Porto Alegre, RS: Artmed.
First, M. B., Spitzer, R. L., Gibbon, M., \& Williams, J. B. W. (1997). Structured Clinical Interview for DSM-IV Axis I Disorders - Clinician version (SCID-CV). Washington, DC: American Psychiatric Press.

Furmark, T. (2000). Social phobia. From epidemiology to brain function. (Unpublished master's thesis). Uppsala University, Sweden.

Hansson, L. (2001, May). Outcome assessment in psychiatric service evaluation. Social Psychiatry and Psychiatric Epidemiology, 36(3), 244-248.

Heimberg, R. G., Dodge, C. S., \& Becker, R. E. (1987). Social phobia. In L. Michelson \& M. L. Ascher, Anxiety and stress disorders (pp. 220-309). New York: Guilford.

Herbert, J. D., Hope, D. A., \& Bellack, A. S. (1992, May). Validity of the distinction between generalized social phobia and avoidant personality disorder. Journal of Abnormal Psychology, 101(2), 332-339.

Osório, F. L. (2008). Transtorno de ansiedade social: Validação de instrumentos de avaliação. (Tese de Doutorado nãopublicada). Faculdade de Medicina, Universidade de São Paulo, Ribeirão Preto, SP.

Pasquali, L., \& Gouveia, V. V. (1990, set./dez.). Escala de Assertividade de Rathus - RAS: Adaptação brasileira. Psicologia: Teoria e Pesquisa, 6(3), 233-249.

Perreault, M., Katerelos, T. E., Sabourin, S., Leichner, P., \& Desmarais, J. (2001). Information as a distinct dimension for satisfaction assessment of outpatient psychiatric services. International Journal of Health Care Quality Assurance, 14(3), 111-120.

Ruggeri, M. (1994). Patients' and relatives' satisfaction with psychiatric services: The state of the art of its measurement. Social Psychiatry and Psychiatric Epidemiology, 29(5), 212-227.

Safren, S. A., Heimberg, R. G., Brown, E. J., \& Holle, C. (19961997). Quality of life in social phobia. Depression and Anxiety, 4(3), 126-133.

Selltiz, C., Wrightsman, L. S., \& Cook, S.W. (1987). Delineamentos de pesquisa: Vol. 1. Métodos de pesquisa nas relações sociais (2. ed.). São Paulo, SP: EPU.

Smith, G. R., Jr., Manderscheid, R. W., Flynn, L. M., \& Steinwachs, D. M. (1997, August). Principles for assessment of patient outcomes in mental health care. Psychiatric Services, 48(8), 1033-1036.

Stein, M. B., \& Kean, Y. M. (2000, October). Disability and quality of life in social phobia: Epidemiologic findings. American Journal Psychiatry, 157(10), 1606-1613.

Stopa, L., \& Clark, D. M. (1993, March). Cognitive processes in social phobia. Behaviour Research and Therapy, 31(3), 255-267.

Wittchen, H. U., \& Beloch, E. (1996, June). The impact of social phobia on quality of life. International Clinical Psychopathology, 11(Suppl. 32), 15-23.

Wittchen, H. U., Fuetsch, M., Sonntag, H., Müller, N., \& Liebowitz, M. (2000, February). Disability and quality of life in pure and comorbid social phobia. Findings from a controlled study. European Psychiatry, 15(1), 46-58. 